\title{
Genome Sequence Resource of a Puccinia striiformis Isolate Infecting Wheatgrass
}

\author{
Yuxiang Li, ${ }^{1}$ Chongjing Xia, ${ }^{1,2}$ Meinan Wang, ${ }^{1}$ Chuntao Yin, ${ }^{1}$ and Xianming Chen ${ }^{1,3, \dagger}$ \\ ${ }^{1}$ Department of Plant Pathology, Washington State University, Pullman, WA 99164-6430, U.S.A. \\ ${ }^{2}$ Wheat Research Institute, School of Life Science and Engineering, Southwest University of Science and \\ Technology, Mianyang, Sichuan 621010, China \\ ${ }^{3}$ United States Department of Agriculture-Agricultural Research Service, Wheat Health, Genetics, and \\ Quality Research Unit, Pullman, WA 99164-6430, U.S.A.
}

\begin{abstract}
Stripe rust caused by Puccinia striiformis is a disastrous disease of cereal crops and various grasses. To date, 14 stripe rust genomes are publicly available, including $13 P$. striiformis $\mathrm{f} . \mathrm{sp}$. tritici and $1 P$. striiformis $\mathrm{f}$. sp. hordei. In this study, one isolate (11-281) of $P$. striiformis collected from wheatgrass (Agropyron cristatum), which is avirulent to most of standard differential genotypes of wheat and barley, was sequenced, assembled, and annotated. The sequences were assembled to a draft genome of $84.75 \mathrm{Mb}$, which is comparable with previously sequenced $P$. striiformis $\mathrm{f}$. $\mathrm{sp}$. tritici and $P$. striiformis $\mathrm{f}$. $\mathrm{sp}$. hordei isolates. The draft genome comprised 381 scaffolds and contained 1,829 predicted secreted proteins. The high-quality draft genome of the isolate is a valuable resource in shedding light on the evolution and pathogenicity of $P$. striiformis.
\end{abstract}

Puccinia striiformis, causing stripe (yellow) rust on wheat, barley, triticale, rye, and various grasses, is a dikaryotic and obligated biotroph belonging to the Basidiomycota (Chen and Kang 2017). Among all released genomes of $P$. striiformis, only two isolates (104E137A- and 93-210) of $P$. striiformis f. sp. tritici (the wheat stripe rust forma specialis) and one isolate (93TX-2) of $P$. striiformis $\mathrm{f}$. sp. hordei (the barley stripe rust forma specialis) were sequenced by long-read sequencing platforms (Schwessinger et al. 2018; Xia et al. 2018a,b). Here, we present the genome of $P$. striiformis isolate 11-281, which was collected from wheatgrass (Agropyron cristatum (L.) Gaertn.), with virulence on only few genotypes among a large number of wheat and barley genotypes tested. Thus, the genome of this isolate is presumed to maintain more avirulence genes corresponding to resistance genes in both wheat and barley than previously sequenced isolates virulent on many wheat or barley genotypes with various resistance genes (Xia et al. 2018a,b). The genomic information of this isolate will advance the understanding of the evolution of host adaptation in $P$. striiformis and may enable the identification of potential avirulence genes in the pathogen.

Isolate 11-281 was originally collected from a wheatgrass plant in Lincoln County, ID in the United States in 2011. This isolate is virulent on wheat genotypes Lemhi, Michigan Amber, PI 478214, and PS 279 and barley genotypes Topper, Abed Binder 12, and Steptoe. However, the isolate was avirulent on Avocet $S$ and the $18 \mathrm{Yr}$ single-gene lines used to differentiate $P$. striiformis $\mathrm{f}$. sp. tritici races and, thus, it was designated as race PSTv-18 of $P$. striiformis $\mathrm{f}$. $\mathrm{sp}$. tritici (Wan and Chen 2014). Among the 12 barley differentials for identifying $P$. striiformis $\mathrm{f} . \mathrm{sp}$.

\section{${ }^{\dagger}$ Corresponding author: X. Chen; xianming.chen@ars.usda.gov}

Mention of trade names or commercial products in this publication is solely for the purpose of providing specific information and does not imply recommendation or endorsement by the U.S. Department of Agriculture. USDA is an equal opportunity provider and employer.

The author(s) declare no conflict of interest.

Accepted for publication 30 April 2019.

This article is in the public domain and not copyrightable. It may be freely reprinted with customary crediting of the source. The American Phytopathological Society, 2019.

\section{Funding}

This study was supported by United States Department of Agriculture-Agricultural Research Service (USDA-ARS project number 2090-22000-018-00D) and Washington Grain Commission (project number 13C-3061-5665).

\section{Keywords}

analytical and theoretical plant pathology 
Table 1. Summary statistic of Puccinia striiformis genome 11-281

\begin{tabular}{lcc} 
& \multicolumn{2}{c}{ Statistics } \\
\cline { 2 - 3 } Feature & Primary & Haplotype \\
Genome size $(\mathrm{Mb})$ & 84.75 & 60.09 \\
Scaffolds & 381 & 873 \\
N50 scaffold length (kb) & 431.12 & 116.17 \\
Contigs & 427 & 873 \\
N50 contig length (kb) & 385.55 & 116.17 \\
GC (\%) & 44.37 & 44.37 \\
Protein-coding genes & 16,869 & 12,145 \\
Total tRNAs: & 667 & 496 \\
rRNA & 32 & 32 \\
Transposable elements (Mb) & 30.34 & 20.85 \\
Transposable elements (\%) & 35.81 & 34.69 \\
Annotation completeness (\%) & 94.16 & 79.40 \\
Secreted protein genes & 1,829 & 1,318 \\
\hline
\end{tabular}

hordei races, the isolate was virulent only on differentials Topper and Abed Binder 12 and, thus, designated as PSH-33 (Wan and Chen 2012). Genomic DNA was extracted from dried urediniospores using the cetyltrimethylammonium bromide method, with modifications (Chen et al. 1993). The genomic DNA was sequenced using both Illumina Hiseq2000 sequencing by Novogene Corporation (Sacramento, CA, U.S.A.) and Pacific BioSciences (PacBio) RS II sequencing by the Genomics Laboratory in Washington State University (Pullman, WA, U.S.A.). Illumina sequencing yielded $6,656,674,305$ bp of sequencing data, an estimated $79 \times$ average coverage. Raw paired-end Illumina sequencing reads were quality checked using FastQC v0.11.6, and low-quality reads were trimmed using Trimmomatic v0.36 (Bolger et al. 2014). Eleven single-molecule real-time cells were used in PacBio sequencing, which yielded $9,366,231,501$ bp of sequencing data, representing an estimated average coverage of $111 \times$. The raw PacBio sequence reads were corrected with short Illumina reads using proovread2.14.0 (Hackl et al. 2014). The error-corrected reads were then assembled to contigs using Falcon, Falcon-unzip, and Canu v1.7 (Chin et al. 2016; Koren et al. 2017). The mapping coverage of every assembled contig was calculated using Qualimap v2.2.1 by mapping Illumina reads to PacBio reads (Okonechnikov et al. 2015). Contigs with a mean coverage greater than $1,000 \times$ were considered as mitochondrial contigs and were excluded from the assembled genomic sequences. The draft assembly contigs were polished using Illumina reads and Pilon1.22 (Walker et al. 2014). The preassembled contigs were scaffolded by SSPACE-STANDARD3.0 and GapFiller v1-10 using paired-ends Illumina reads (Boetzer et al. 2010). The sequences assembled by Falcon-unzip and Canu assembler were merged to form a single superior sequence using Metassembler v1.5 (Wences and Schatz 2015). The merged sequences were then aligned to those of the three $P$. striiformis isolates sequenced by PacBio using the nucmer script in the MUMmer 3.0 package (Kurtz et al. 2004). The contigs in alignment with the reference scaffold were manually merged into one scaffold. The assembled $P$. striiformis 11-281 genome consists of 381 primary and 873 haplotype scaffolds, with the genome size of 84.75 and $60.09 \mathrm{Mb}$, respectively (Table 1). The genome size of primary scaffold is comparable with those of isolates 104E137A- (83.36 Mb) (Schwessinger et al. 2018), 93-210 (84.63 Mb), and 93TX-2 (77.39 Mb) (Xia et al. 2018a). The average nucleotide identity (ANI) was calculated between isolate 11-281 and each of the three reference genomes (Jain et al. 2018). All ANI values were above $98.8 \%$, indicating the high genomic similarities of different $P$. striiformis isolates.

Total RNA was extracted separately from urediniospores, germinated urediniospores, and leaves 24 h, 48 h, 7 days, and 14 days after inoculation on susceptible and resistant wheat cultivars using TRIzol Reagent with the PureLink RNA Mini Kit (Invitrogen, San Diego, CA, U.S.A.). Equal amounts of the 10 RNA samples were mixed for mRNA sequencing by Illumina Hiseq2000 sequencing (Novogene Corporation, Sacramento, CA, U.S.A.). Error-corrected mRNA sequencing reads were aligned to the assembled genome using HISAT2 v2.1.0 (Kim et al. 2015). The alignments were assembled into transcripts by StringTie v1.3.4 (Pertea et al. 2015). Gene predictions by the Ab initio method were conducted using Augustus v3.2.3, GeneMark v3.54, and SNAP (Korf 2004; Ter-Hovhannisyan et al. 2008). Repeats were masked using RepeatMasker v4.0.7 and RepeatModeler v1.0.9. tRNAs were predicted using tRNAscan v2.0 (Lowe and Chan 2016). All predicted genes were incorporated into the annotation pipeline MAKER2 v2.31.10 (Holt and Yandell 2011). The assembled transcripts by StringTie were used in the analysis with MAKER2 to improve the quality of genome annotation. In total, 16,869 and 
12,145 protein-coding genes were identified from the primary and haplotype scaffolds, respectively (Table 1). Of these genes, 8,951 (53.06\%) and 6,897 (56.79\%) were validated by the assembled transcripts using Gffcompare v0.10.6 and StringTie v1.3.4, respectively. The genome annotation completeness was assessed by benchmark universal single-copy orthologs (BUSCO v2.0.1) (Simão et al. 2015). The 1,298 complete and fragmented BUSCOs from the primary scaffolds and haplotigs were detected from 1,335 BUSCOs in the dataset of basidiomycetes, with the overall completeness of $97.22 \%$. Of the 16,869 protein-coding genes in the primary scaffold, 1,957 and 1,415 signal peptides were identified by SignalP 4.1 from the primary set and the haplotype set of protein-coding genes (Nielsen 2017), respectively, and 1,829 with signal peptides and 1,318 proteins with excluded transmembrane helices were predicted to be secreted proteins (Table 1).

Because grasses harbor a higher diversity of genetic variation among isolates than wheat and barley hosts, they are reservoirs of genetic variation of $P$. striiformis (Cheng et al. 2016). Some $P$. striiformis isolates infecting wheatgrasses could be the origin of different formae speciales. The genome sequence of isolate 11-281 presented here is an important resource, which will make it possible to identify forma specialis-specific genes and avirulence genes in the future by comparable genomics analyses with other $P$. striiformis isolates that have different virulence spectra.

\section{Accession Number}

The assembled genome sequence of $P$. striiformis isolate 11-281 has been deposited at GenBank under the accession number SBIN00000000. The version described in this article is version SBIN01000000. The raw data for this experiment have been submitted to NCBI with SRA study SRR8446446. More information of annotation files and protein sequences are available on GitHub at https://github.com/yuxiang-li/Puccinia_striiformis_genome_11_281.

\section{Acknowledgments}

We thank Washington State University Kamiak High Performance Computing for the computing resources and technique support and The China Scholarship Council for its scholarship to $\mathrm{Y}$. Li.

\section{Literature Cited}

Boetzer, M., Henkel, C. V., Jansen, H. J., Butler, D., and Pirovano, W. 2010. Scaffolding pre-assembled contigs using SSPACE. Bioinformatics 27:578-579.

Bolger, A. M., Lohse, M., and Usadel, B. 2014. Trimmomatic: A flexible trimmer for Illumina sequence data. Bioinformatics 30:2114-2120.

Chen, X. M., and Kang, Z. S. 2017. Introduction: History of research, symptoms, taxonomy of the pathogen, host range, distribution, and impact of stripe rust. Pages 1-33 in: Stripe Rust. X. M. Chen and Z. S. Kang, eds. Springer, Dordrecht, The Netherlands.

Chen, X. M., Line, R. F., and Leung, H. 1993. Relationship between virulence variation and DNA polymorphism in Puccinia striiformis. Phytopathology 83:1489-1497.

Cheng, P., Chen, X. M., and See, D. 2016. Grass hosts harbor more diverse isolates of Puccinia striiformis than cereal crops. Phytopathology 106:362-371.

Chin, C.-S., Peluso, P., Sedlazeck, F. J., Nattestad, M., Concepcion, G. T., Clum, A., Dunn, C., O'Malley, R., Figueroa-Balderas, R., Morales-Cruz, A., Cramer, G. R., Delledonne, M., Luo, C., Ecker, J. R., Cantu, D., Rank, D. R., and Schatz, M. C. 2016. Phased diploid genome assembly with single-molecule real-time sequencing. Nat. Methods 13:1050-1054.

Hackl, T., Hedrich, R., Schultz, J., and Förster, F. 2014. proovread: Large-scale highaccuracy PacBio correction through iterative short read consensus. Bioinformatics 30:3004-3011.

Holt, C., and Yandell, M. 2011. MAKER2: An annotation pipeline and genomedatabase management tool for second-generation genome projects. BMC Bioinf. 12:491.

Jain, C., Rodriguez-R, L. M., Phillippy, A. M., Konstantinidis, K. T., and Aluru, S. 2018. High throughput ANI analysis of $90 \mathrm{~K}$ prokaryotic genomes reveals clear species boundaries. Nat. Commun. 9:5114.

Kim, D., Langmead, B., and Salzberg, S. L. 2015. HISAT: A fast spliced aligner with low memory requirements. Nat. Methods 12:357-360.
Koren, S., Walenz, B. P., Berlin, K., Miller, J. R., Bergman, N. H., and Phillippy, A. M. 2017. Canu: Scalable and accurate long-read assembly via adaptive $k$-mer weighting and repeat separation. Genome Res. 27:722-736.

Korf, I. 2004. Gene finding in novel genomes. BMC Bioinf. 5:59.

Kurtz, S., Phillippy, A., Delcher, A. L., Smoot, M., Shumway, M., Antonescu, C., and Salzberg, S. L. 2004. Versatile and open software for comparing large genomes. Genome Biol. 5:R12.

Lowe, T. M., and Chan, P. P. 2016. tRNAscan-SE On-line: Integrating search and context for analysis of transfer RNA genes. Nucleic Acids Res. 44:W54-W57.

Nielsen, H. 2017. Predicting secretory proteins with SignalP. Pages 59-73 in: Protein Function Prediction: Methods and Protocols. D. Kihara, ed. Springer, Dordrecht, The Netherlands.

Okonechnikov, K., Conesa, A., and García-Alcalde, F. 2015. Qualimap 2: Advanced multi-sample quality control for high-throughput sequencing data. Bioinformatics 32:292-294.

Pertea, M., Pertea, G. M., Antonescu, C. M., Chang, T. C., Mendell, J. T., and Salzberg, S. L. 2015. StringTie enables improved reconstruction of a transcriptome from RNA-seq reads. Nat. Biotechnol. 33:290-295.

Schwessinger, B., Sperschneider, J., Cuddy, W. S., Garnica, D. P., Miller, M. E., Taylor, J. M., Dodds, P. N., Figueroa, M., Park, R. F., and Rathjen, J. P. 2018. A near complete haplotype-phased genome of the dikaryotic wheat stripe rust fungus Puccinia striiformis f. sp. tritici reveals high interhaplotype diversity. MBio 9: e02275-17.

Simão, F. A., Waterhouse, R. M., Ioannidis, P., Kriventseva, E. V., and Zdobnov, E. M. 2015. BUSCO: Assessing genome assembly and annotation completeness with single-copy orthologs. Bioinformatics 31:3210-3212.

Ter-Hovhannisyan, V., Lomsadze, A., Chernoff, Y. O., and Borodovsky, M. 2008. Gene prediction in novel fungal genomes using an ab initio algorithm with unsupervised training. Genome Res. 18:1979-1990. 
Walker, B. J., Abeel, T., Shea, T., Priest, M., Abouelliel, A., Sakthikumar, S., Cuomo, C. A., Zeng, Q., Wortman, J., Young, S. K., and Earl, A. M. 2014. Pilon: An integrated tool for comprehensive microbial variant detection and genome assembly improvement. PLoS One 9:e112963.

Wan, A. M., and Chen, X. M. 2012. Virulence, frequency, and distribution of races of Puccinia striiformis $\mathrm{f}$. $\mathrm{sp}$. tritici and $P$. striiformis $\mathrm{f}$. $\mathrm{sp}$. hordei identified in the United States in 2008 and 2009. Plant Dis. 96:67-74.

Wan, A. M., and Chen, X. M. 2014. Virulence characterization of Puccinia strifformis $\mathrm{f}$. sp. tritici using a new set of $Y r$ single-gene line differentials in the United States in 2010. Plant Dis. 98:1534-1542.
Wences, A. H., and Schatz, M. C. 2015. Metassembler: Merging and optimizing de novo genome assemblies. Genome Biol. 16:207.

Xia, C., Wang, M., Yin, C., Cornejo, O. E., Hulbert, S. H., and Chen, X. 2018a. Genome sequence resources for the wheat stripe rust pathogen (Puccinia striiformis $f$. sp. tritici) and the barley stripe rust pathogen (Puccinia strifformis f. sp. hordei). Mol. Plant-Microbe Interact. 31:1117-1120.

Xia, C., Wang, M., Yin, C., Cornejo, O. E., Hulbert, S. H., and Chen, X. 2018b. Genomic insights into host adaptation between the wheat stripe rust pathogen (Puccinia striiformis f. sp. triticl) and the barley stripe rust pathogen (Puccinia striiformis f. sp. hordei). BMC Genomics 19:664. 